\title{
Staging of the Axilla in Breast Cancer and the Evolving Role of Axillary Ultrasound
}

\author{
Michael Y Chen' \\ William E Gillanders ${ }^{1,2}$ \\ 'Department of Surgery, Washington \\ University, St Louis, MS, USA; ${ }^{2}$ Siteman \\ Cancer Center in St. Louis, St Louis, \\ MS, USA
}

\begin{abstract}
Axillary lymph nodes have long been recognized as a route for breast cancer to spread systemically. As a result, staging of the axilla has always played a central role in the treatment of breast cancer. Anatomic staging was believed to be important for two reasons: 1) it predicts prognosis and guides medical therapy, and 2) it is a potential therapy for removal of disease in the axilla. This paradigm has now been called into question. Prognostic information is driven increasingly by tumor biology, and trials such as the ACOSOG Z0011 demonstrates removal of axillary disease is not therapeutic. Staging of the axilla has undergone a dramatic de-escalation; however, sentinel lymph node biopsy (SLNB) is still an invasive surgery and represents a large economic burden on the healthcare system. In this review, we outline the changing paradigms of axillary staging in breast cancer from emphasis on anatomic staging to tumor biology, and the evolving role of axillary ultrasound, bringing patients less invasive and more personalized therapy.
\end{abstract}

Keywords: breast cancer, axillary staging, SLNB, ALND, axillary ultrasound, Z0011

\section{Introduction to Staging of the Axilla in Breast Cancer}

Breast cancer is the most prevalent cancer in women, and the second-leading cause of cancer death. In 2021, it is estimated that 280,000 cases of breast cancer will be diagnosed in the US, resulting in over 43,000 deaths. ${ }^{1}$ Axillary surgery has long been central to the staging and treatment of breast cancer. In the 19th century, Rudolph Virchow postulated that axillary lymph node metastases facilitated the distant metastatic spread of breast cancer. $^{2}$ This led William Halsted to propose radical mastectomy (excision of the breast tissue, pectoralis muscle, and ipsilateral axillary lymph nodes) for the treatment of breast cancer. ${ }^{3}$ In the century since Halsted pioneered the radical mastectomy, advances in diagnostic imaging, systemic therapy, radiation therapy and an improved understanding of breast cancer biology have led to a dramatic deescalation of breast cancer surgery. ${ }^{4}$ The modified radical mastectomy replaced the radical mastectomy. Breast conserving surgery further decreased the invasiveness of breast cancer surgery. Sentinel lymph node mapping made possible sentinel lymph node biopsy (SLNB) for early-stage breast cancers, sparing patients the morbidity of axillary lymph node dissection (ALND). ${ }^{5,6}$ Recent advances in axillary imaging and molecular profiling of primary breast cancers have called into question the necessity of SLNB/axillary surgery. Axillary ultrasound (AUS) has been proposed as a non-invasive and cost-effective
Correspondence: William E Gillanders Siteman Cancer Center in St. Louis, 660 South Euclid Avenue Campus Box 8109, St Louis, MS, 63II0, USA

$\mathrm{Tel}+$ I 3 | 47470072

Email gillandersw@wustl.edu 
alternative to SLNB for staging the axilla in breast cancer. Here, we review evolving paradigms for staging of the axilla in breast cancer, with a particular emphasis on the current and future role of axillary ultrasound.

\section{Implications for Medical Decision Making}

Axillary surgery has two potential roles in breast cancer management. First, it provides anatomic staging information which is used to predict prognosis and inform medical decision making related to adjuvant therapy. Second, it is a potential therapy for management of disease in the axilla. Both of these roles have been called into question, leading to an ongoing reevaluation of the necessity of axillary surgery.

The presence of axillary lymph node metastases has historically been considered an important prognostic factor in breast cancer, and impacted many aspects of medical decision making. Anatomic staging of breast cancer includes both clinical and pathological staging. ${ }^{7}$ Clinical staging integrates physical examination and imaging findings, while pathological staging incorporates the results of surgical pathology. In the AJCC TNM staging schema, metastatic disease in $1-3,4-9$, and $\geq 10$ axillary lymph nodes constitutes N1, N2, and N3 diseases, respectively. Greater nodal involvement is associated with a worse prognosis, suggesting that more extensive surgery, radiation, and systemic therapy may be beneficial. ${ }^{8}$

With improved understanding of tumor biology, medical decision-making is increasingly dependent on molecular profiling of the primary tumor. Biomarker and gene expression profiles are now routinely used to predict prognosis and response to therapy. Biomarker profiles are a surrogate for molecular subtype, and increasingly drive neoadjuvant and adjuvant systemic therapy decisions. Biomarker profile is particularly important for decisions regarding the use of targeted therapies, such as endocrine therapy, or therapy with HER2-targeting agents. Molecular profiling with tests, such as Oncotype Dx, provides additional prognostic information and is often more informative than anatomic staging information for predicting response to therapy. ${ }^{9,10}$

Recent basic science studies using next-generation sequencing technologies confirm that most breast cancer metastases arise from the primary tumor rather than lymph node metastases. ${ }^{11}$ This is consistent with the hypothesis that the process of acquiring the biologic ability to metastasize (as outlined in the classic hallmarks of cancer) typically takes place at the primary tumor, not at lymph node metastases. ${ }^{12-14}$

\section{Therapeutic Considerations}

As noted above, clinical decision-making paradigms have evolved in breast cancer and anatomic staging has decreased in importance in terms of medical decisionmaking. In addition, the therapeutic benefit of axillary surgery appears to be limited. The ACOSOG Z0011 trial randomized women with clinical T1-T2 N0 breast cancer and 1-2 positive sentinel lymph nodes at the time of SLNB to no further axillary surgery or completion ALND. Z0011 demonstrated no local control or survival advantage with completion ALND, suggesting that the therapeutic benefit of axillary surgery is minimal or nonexistent. ${ }^{15}$ Similar results were obtained in the IBSCG 23-01 trial. ${ }^{16}$ These trials have led to a paradigm shift in the perceived role of axillary surgery. Axillary surgery is now considered to be primarily a staging procedure, with minimal to no therapeutic benefit. ${ }^{17,18}$ This is reflected in the 16th St. Gallen International Consensus Guidelines in 2019 which states completion ALND is not indicated if patient receives post-lumpectomy radiation and appropriate systemic adjuvant therapy in early-stage breast cancer. $^{19}$

\section{Current Modalities for Axillary Staging Physical Examination}

Axillary staging in newly diagnosed breast cancer begins with physical examination. However, physical examination has consistently been shown to lack sensitivity and specificity. Physical examination lacks the sensitivity for detection of microscopic or even subcentimeter axillary lymph node metastases. Specificity is complicated by the inability to distinguish reactive lymph nodes from lymph node metastases. Various studies have found that the positive predictive value ranges from 61 to $84 \%$, and negative predictive value from 50 to $62 \% .^{20-22}$ Additional testing is required to accurately stage the axilla.

\section{Axillary Ultrasound}

Axillary ultrasound (AUS) is the most commonly used imaging modality for evaluation of the axilla in breast cancer. The evaluation is typically performed using a high frequency $(7.5-17 \mathrm{MHz})$ linear-array transducer with the patient in supine oblique position, arm abducted 
and externally rotated with hand above head ("bathing beauty" position). ${ }^{23}$ AUS is non-invasive, affordable, and leverages readily available technologies. AUS has a PPV of $56-90 \%$ and NPV of $76-84 \% .^{22,24}$ Like all ultrasound technologies, however, it is limited by operator dependency and patient body habitus.

\section{Sentinel Lymph Node Biopsy}

Historically, axillary lymph node dissection (ALND) was performed routinely, providing staging information. In the 1990s, several randomized controlled trials demonstrated no difference in locoregional control, disease free survival, and overall survival between SLNB and ALND in patients with a negative SLNB. ${ }^{25,26}$ Furthermore, SLNB was associated with better quality of life, upper extremity function, and fewer side effects, such as chronic pain, lymphedema, and sensory deficits. ${ }^{5,6,27,28}$ In early-stage breast cancer, SLNB has become the standard of care for staging of the axilla. A meta-analysis of 69 trials with over 8000 patients found SLNB accurately mapped the sentinel node in $96 \%$ of patients with an average false negative rate of $7.3 \%{ }^{29}$

\section{Axillary Lymph Node Dissection}

ALND is now typically reserved for patients with significant axillary disease. It is primarily considered a therapeutic strategy in this context. In the aftermath of ACOSOG Z0011 and IBSCG 23-01 trial, ALND is still indicated for patients with three or more metastatic lymph nodes. ${ }^{30}$ ALND is also indicated in those with less than three positive nodes who either refuse or are not candidates for adjuvant radiation. Since the Z0011 trial excluded patients with extranodal extension, ALND is still performed for patients with less than 3 positive SLNs but with $>2 \mathrm{~mm}$ extranodal extension as up to $33 \%$ may have more extensive disease involving more than 4 nodes. ${ }^{31}$ As mentioned previously, ALND is associated with significant morbidity and thus patient selection must be carefully considered.

\section{Biologic Staging}

Recent decades have seen a dramatic acceleration in the understanding of breast cancer biology and the concomitant development of targeted therapeutics. Biomarker profile and gene expression profiling assays, such as OncotypeDx, are currently used to drive clinical decision making. The increasing role of tumor biology is reflected in the 8th edition of the AJCC staging system. The AJCC system now combines anatomic staging information with HER2 and hormone receptor status to create a clinical prognostic stage. A pathologic prognostic stage was further created with the addition of histologic grade and OncotypeDx $R S$ score. $^{7}$ The revised AJCC staging system more accurately predicts prognosis by integrating both anatomic and biologic staging information. The revised AJCC staging system was derived from over 300,000 patients enrolled in the National Cancer Database between 2010 and 2012 representing over $70 \%$ of breast cancers diagnosed in the US. ${ }^{32}$ However, the prognostic validity of the revised AJCC system assumes availability and access to recommended adjuvant therapy. For example, HER $2^{+}$tumors are downstaged because there is an effective targeted therapy. Similarly, the incorporation of OncotypeDx $R S$ score assumes patients will be managed appropriately.

\section{Current Staging Algorithms}

The current algorithm for staging of the axilla in early stage (T1-2) breast cancer starts with clinical evaluation based on physical exam and imaging (summarized in Figure 1). Clinically node-negative patients typically undergo SLNB. Patients with negative sentinel nodes or only micrometastases do not need further axillary surgery.

Clinically node-positive patients are candidates for ultrasound-guided FNA or core needle biopsy (CNB). In the event of a negative FNA/CNB, SLNB is indicated. If FNA/CNB is positive, ALND is indicated unless neoadjuvant therapy is performed. In patients with positive FNA/ CNB who subsequently receive neoadjuvant chemotherapy and become clinically node negative, SLNB may be considered.

\section{Axillary Ultrasound Characteristics of Abnormal Lymph Nodes on Ultrasound}

AUS can identify disease in ALN based on the presence or absence of defined sonographic criteria, such as absent or infiltrated hilum, or focal or diffuse cortical thickening (Figure 2). ${ }^{33-36}$ Normal lymph nodes in ultrasound have uniform hypoechoic cortex, central fatty hilum, and smooth margins. ${ }^{37}$ Any deviations from this normal appearance are potential signs of metastasis. A cortical thickness of greater than $3 \mathrm{~mm}$ is associated with more than three-fold increase in metastatic disease, loss of fatty hilum is associated with a 27 -fold increase, 


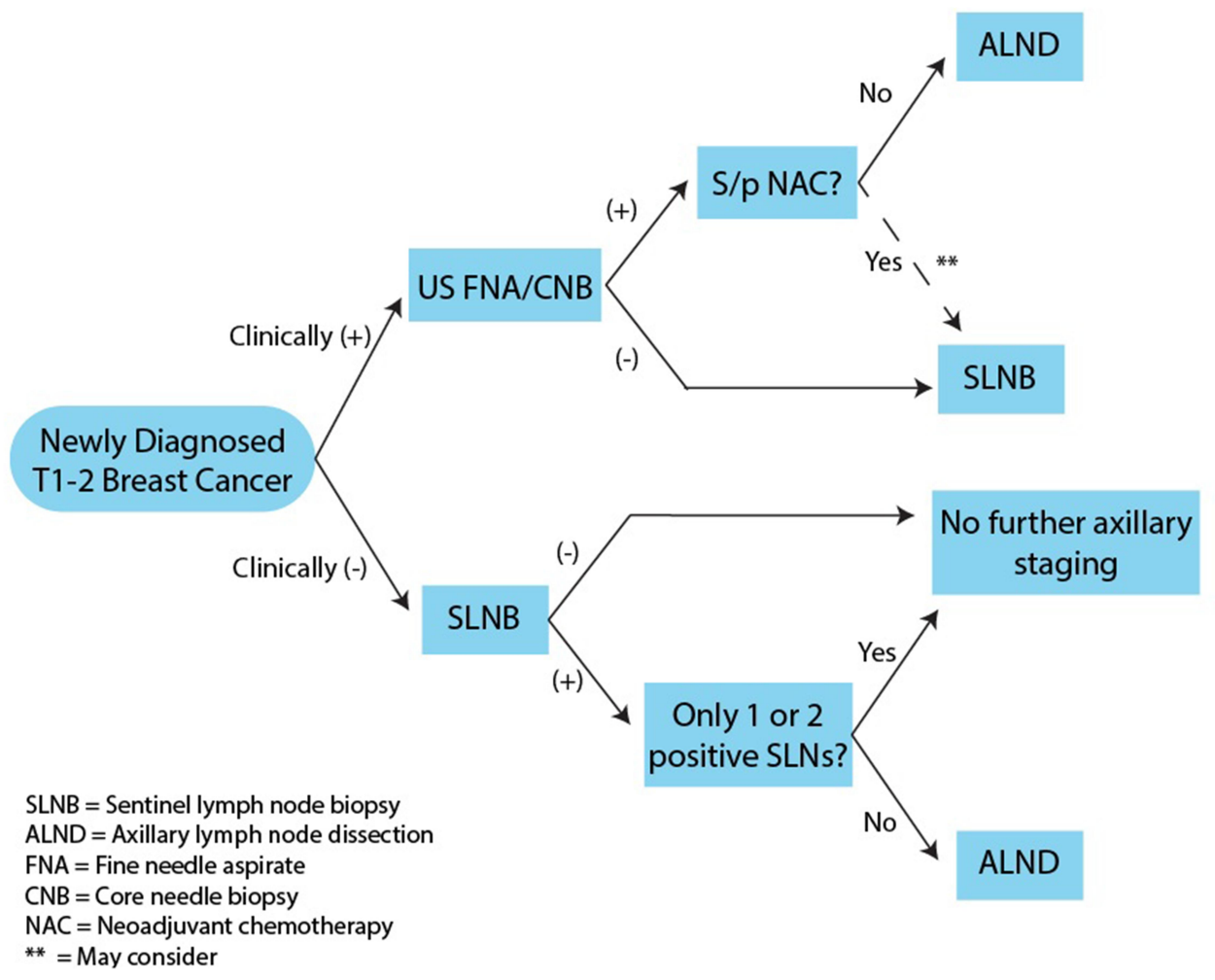

Figure I Overview of current algorithms for axillary staging in early stage breast cancer. SLNB is recommended for patients with a clinically negative axilla. If $\geq 3$ sentinel nodes are positive, completion ALND is recommended. No further intervention is indicated for $\leq 2$ sentinel nodes are positive. US guided FNA/CNB is recommended for patients with a clinically positive axilla. If FNA/CNB is positive, ALND is indicated. If FNA/CNB is negative, SLNB \pm ALND is recommended. For patients with a positive FNA/CNB who undergo NAC, SLNB may be considered depending on response to treatment.

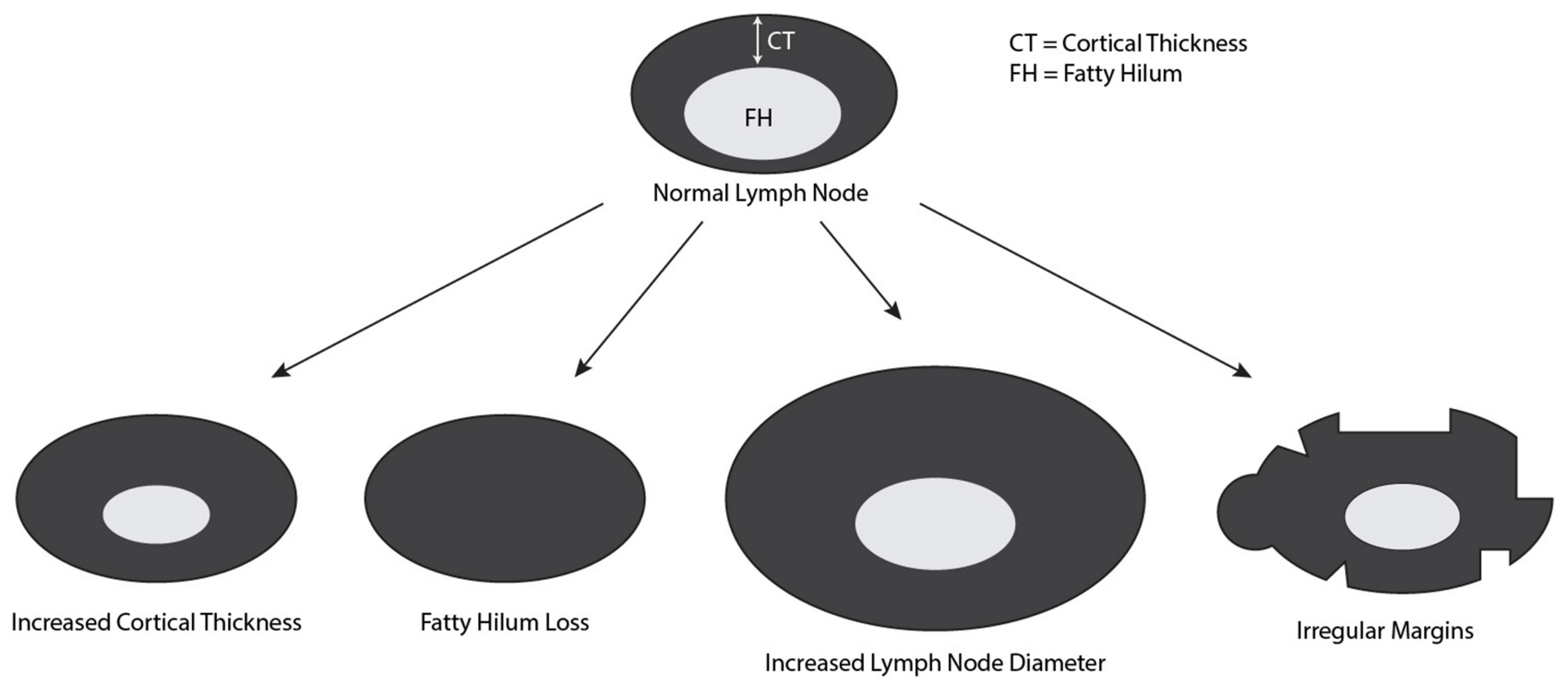

Figure 2 Ultrasound features suggesting metastatic involvement of axillary lymph nodes. Normal lymph nodes have a uniform hypoechoic cortex, central fatty hilum, and smooth margins. Features identified to be predictive of metastatic lymph node disease include increased cortical thickness $(O R=3)$, fatty hilum loss $(O R=27)$, increased diameter, and irregular margins $(\mathrm{OR}=3)$. While the features high odds ratios, they are not individually predictive of lymph node metastasis and have poor AUCs.

and tumor margin irregularity is associated with a 3-fold increase. ${ }^{38,39}$ Lymph node diameter greater than $10 \mathrm{~mm}$ is also associated with increased risk of metastatic disease. While these features have high odds ratios, individually they have poor AUC in predicting the presence of metastatic disease. 


\section{Axillary Ultrasound at Initial Presentation}

Since physical examination is neither sensitive nor specific in terms of predicting the likelihood of nodal metastasis, axillary ultrasound (AUS) is an important non-invasive adjunct to the assessment of the axilla on initial presentation. Currently, AUS is most informative for the evaluation of patients with clinically palpable nodes. ${ }^{8}$ This offers the opportunity to perform FNA or core biopsy on suspicious nodes, helping to identify candidates for ALND rather than SLNB to streamline care. The ability of US FNA to predict disease was shown to have a sensitivity and specificity of $79.6 \%$ and $98.3 \%$, respectively, in a meta-analysis by Houssami et al. ${ }^{40}$

The role of AUS in the pre-operative evaluation of the axilla has been called into question since the ACOSOG Z0011 trial. Some have argued that a positive AUS would unnecessarily commit many patients to ALND who met criteria for observation in the Z0011 trial, namely T1-2 tumors with 1-2 positive SLNs. However, there is evidence that disease identified by AUS FNA suggests a higher axillary disease burden compared to the disease identified by SLNB. Positive AUS FNA is associated with more positive nodes, larger tumors, higher tumor grades, extranodal extension, lymphovascular invasion, and distant metastasis. ${ }^{41,42}$ Several case series have found that only $3-5 \%$ of patients with positive AUS FNA met Z0011 criteria and could have avoided ALND, thus streamlining axillary surgical care for $28.6 \%$ of nodepositive patients. ${ }^{43,44}$ This suggests that AUS continues to play a vital role in the pre-operative evaluation in newly diagnosed breast cancer.

\section{Axillary Ultrasound After Neoadjuvant Chemotherapy}

Historically, patients with node-positive disease underwent ALND after neoadjuvant chemotherapy (NAC). However, this approach is morbid, and $40 \%$ and $70 \%$ of patients convert from node-positive to node-negative disease following NAC and targeted therapy, respectively. ${ }^{45}$ Several prospective studies have looked into the efficacy of SLNB post-NAC. In the NSABP B27, SENTINA, ACOSOG Z1071, and SN FNAC trials, SLNB was limited by high false negative rates of $10.7 \%, 14.2 \%, 12.6 \%$ and $8.4 \%$, respectively. ${ }^{45}$ Patients with a suspicious AUS were node positive $71.8 \%$ of the time compared to $56.5 \%$ for patients with a normal AUS. This suggests a role for AUS evaluation in order to guide post-NAC surgical management of the axilla. In a retrospective comparison of pre- and postNAC imaging, AUS had a sensitivity of $69.8 \%$ for detecting persistent LN metastasis, which outperformed MRI and PET-CT. ${ }^{46}$ Furthermore, the utility of axillary ultrasound may depend on the molecular subtype of the cancer. ${ }^{47}$ Di Micco et al found PPV to be $100 \%$ and $70.5 \%$ for luminal A and luminal B breast cancer, respectively, and NPV to be $93.3 \%$ for both HER $2^{+}$and triple negative breast cancers. Thus, AUS could potentially decide the need for ALND when used as part of a clinical decision algorithm that incorporates tumor biology.

As outlined in section 2.6, all patients with clinically positive axilla should undergo ultrasound evaluation. This serves not only as an opportunity for biopsy but also as a baseline imaging comparison in case NAC is used. The use of AUS in restaging of the axilla post-NAC varies by institution. When used, patients with previously clinically positive axilla who have downstaged to $\mathrm{cN} 0$ after NAC undergo AUS. If AUS demonstrates no persistent disease, patient is eligible for SLNB instead of ALND (Figure 3).

\section{Axillary Ultrasound vs Sentinel Lymph Node Biopsy Limitations of Sentinel Lymph Node Biopsy}

Although the sentinel lymph node hypothesis is elegant, SLNB has important limitations suggesting that alternative approaches to axillary staging should be considered. First, SLNB is an invasive procedure, but is not considered

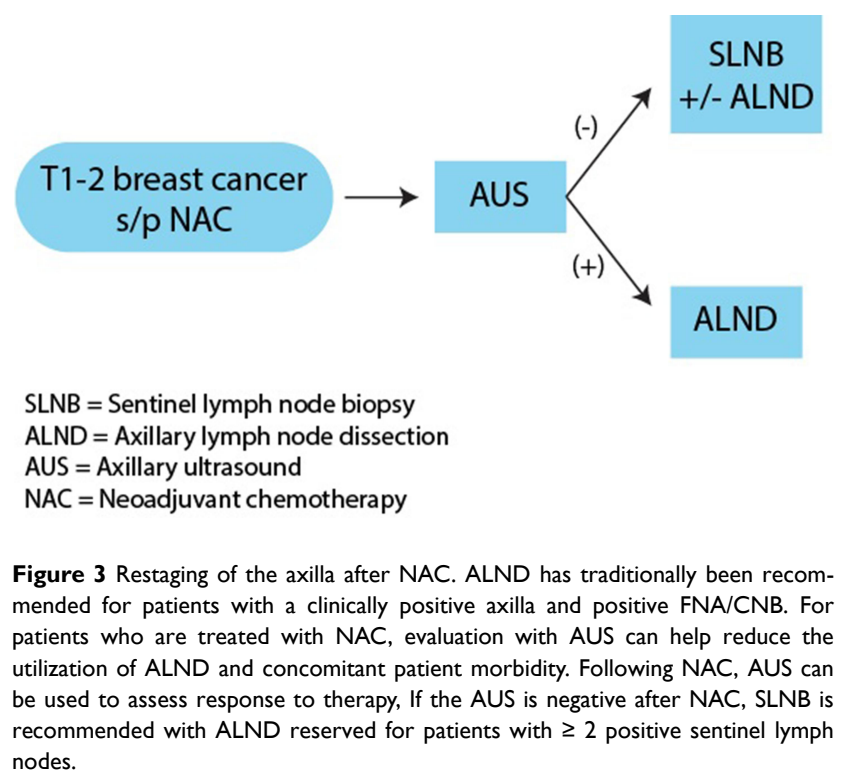


therapeutic. Second, the importance of anatomic staging information is eroding in an era where biomarker profile and gene expression profile are driving medical oncology decision making. Finally, SLNB represents an enormous investment in health care resources.

SLNB is an invasive surgical procedure with significant risks. Large prospective trials, such as ACOSOG Z0010, NSABP B-32, and the ALMANAC trial, have documented SLNB complications including allergic reactions to isosulfan blue dye $(0.1-1.0 \%)$, wound infection (1.0-10\%), seroma (7.1\%), paresthesias (8.6-11\%), and hematoma $(1.4 \%){ }^{26,27,48,49}$ For many breast cancer patients, SLNB is the most morbid component of breast conserving surgery. Of note, not all SLNBs are successful. In $3-5 \%$ of cases, the SLNB cannot be identified, in which case current guidelines recommend that an ALND be performed. ${ }^{30}$ ALND is a morbid procedure with no therapeutic benefit in patients with clinical N0 disease.

Furthermore, SLNB currently represents an enormous investment in healthcare resources. Henry-Tillman et al found charges associated with SLNB at University of Arkansas to total $\$ 23,956 .^{50}$ Based on this number, we estimate that the health care system invests approximately $\$ 3$ billion dollars annually on an invasive surgical procedure that is not considered therapeutic, and has limited impact on medical oncology and radiation oncology decision making. McEvoy et al have conducted cost-effective analyses in $\mathrm{T} 1-2 \mathrm{~N} 0, \mathrm{HR}^{+} / \mathrm{HER} 2^{-}$breast cancer patients, demonstrating observation was superior to SLNB with net monetary benefit of $\$ 655,659$ for observation vs $\$ 641,778$ for SLNB. This suggests that the benefit of observation is not only economic but also reflected in improved quality of life. $^{51}$

\section{Evolving Paradigms: Biologic vs Anatomic Staging}

In the post-Z0011 era, the centrality of surgical axillary staging to the management of breast cancer is being questioned. First, axillary surgery is not considered therapeutic. The ACOSOG Z0011 trial randomized women with clinical T1-T2 N0 breast cancer and a positive SLNB to no further axillary surgery or to completion ALND, and demonstrated no local control or survival advantage with completion ALND. Similar results were obtained in the IBSCG 23-01 trial. ${ }^{15,16}$ Second, the importance of anatomic staging information provided by SLNB is decreasing in importance. Decisions regarding adjuvant therapy are complex, and integrate a significant amount of clinical and pathologic information, such as age, performance status, biomarker profile, and gene expression profile. ${ }^{52-55}$ Although anatomic staging is important, it is decreasing in importance as a driver of medical decision making and this reality is beginning to be reflected in clinical guidelines.

Systemic therapy is increasingly driven by tumor biology, such as gene expression and receptor status. For instance, HER $2^{+}$breast cancers are at least considered for systemic chemotherapy regardless of nodal status. ${ }^{8}$ For $\mathrm{HR}^{+} / \mathrm{HER} 2^{-}$cancers, chemotherapy is determined by nodal status only if there are $\geq 4$ positive nodes, with gene expression assays used to determine the need for chemotherapy for cancers with less than 4 positive nodes. Finally, in triple negative breast cancer, chemotherapy is considered regardless of nodal status.

Adjuvant locoregional therapy is still driven by anatomical staging. Guidelines recommend additional regional radiotherapy when there are axillary lymph node metastases. ${ }^{8}$ However, this is increasingly called into question for early-stage breast cancer. ${ }^{56-58}$ Whelan et al, in a trial where women with node-positive or high-risk node-negative breast cancers were randomized to whole-breast radiation vs whole-breast radiation plus regional nodal radiation, found reduced recurrence but no improvement in overall survival after 10 years with nodal radiation. ${ }^{57}$ This suggests that future locoregional therapy should not only take into account anatomic staging but also tumor biology.

\section{Current Trials Comparing AUS to SLNB}

The feasibility of AUS as an independent staging modality is currently being explored. In a prospective pilot study, Cyr et al demonstrated AUS to be a promising noninvasive alternative to SLNB in patients with early-stage breast cancer. ${ }^{59}$ Three large clinical trials are currently underway to investigate the role of AUS in staging of the axilla in patients with early-stage breast cancer undergoing breast conservation surgery (BCS) with a clinically negative axilla (Table 1). These studies have the potential to dramatically alter the paradigm for axillary staging as the unifying hypothesis for these studies is that most patients with a negative AUS do not require additional axillary staging.

\section{SOUND Trial}

The SOUND Trial (Sentinel node vs Observation after axillary UltrasouND) is a prospective randomized 


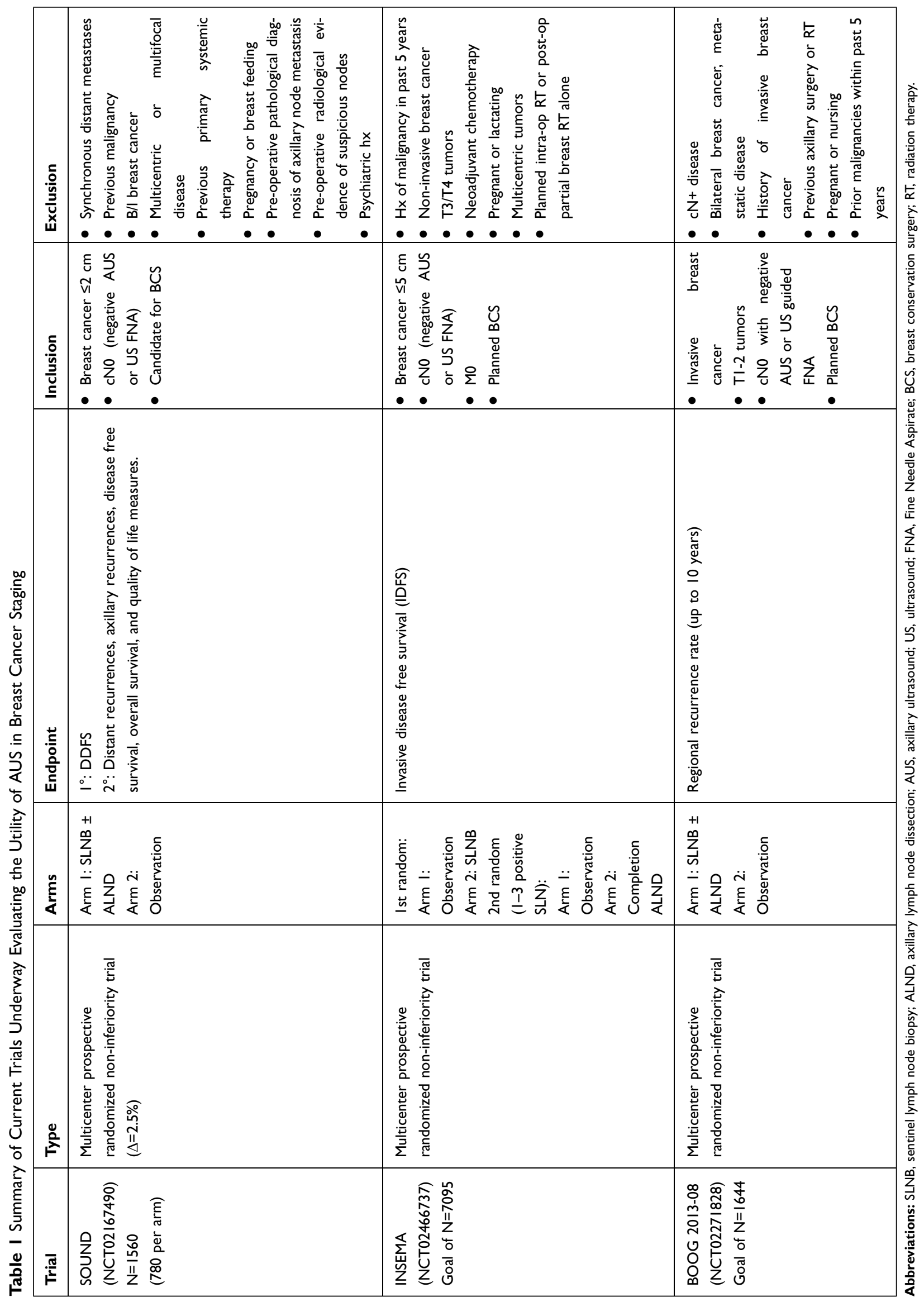


multicenter non-inferiority trial designed by the European Institute of Oncology of Milan (NCT02167490). Inclusion criteria include breast cancer $\leq 2 \mathrm{~cm}$, clinically negative axilla with negative AUS or US guided FNA, and candidate for BCS. The trial has recruited 1560 patients who were randomized to either SLNB \pm ALND or observation. The primary endpoint is distant-disease-free survival. Secondary endpoints are incidence of distant recurrences, axillary recurrences, disease-free survival, overall survival, and quality of life measures.

\section{INSEMA Trial}

Another trial underway is the multicenter German/Austrian Intergroup-Sentinel-Mamma (INSEMA) trial (NCT0246 6737). It is a prospective randomized non-inferiority trial with the goal of enrolling 7095 patients. The trial has two randomizations. The first will be to have no axillary surgery or SLNB. Patients who have undergone SLNB with 1-3 macrometastases in sentinel lymph nodes will undergo a second randomization for either no further axillary surgery or completion ALND. Inclusion criteria are women with breast cancer $\leq 5 \mathrm{~cm}$, $\mathrm{cN} 0$ with negative AUS or US FNA, no evidence of metastasis, and planned BCS. Patients with a history of malignancy within last 5 years, non-invasive breast cancer, T3/T4 tumors, NAC, pregnant or lactating, multicentric tumors, and planned intraoperative radiotherapy (eg Intrabeam) or postoperative partial breast irradiation (eg multicatheter technique) alone are excluded. The primary outcome is disease-free survival after breast conservation surgery.

\section{BOOG 2013-08}

Finally, the Dutch BOOG 2013-08 (NCT02271828) is another non-inferiority multicenter randomized control trial that will randomize patients to observation or SLNB \pm ALND. Inclusion criteria include pathologically confirmed invasive breast cancer, T1-2 tumors, $\mathrm{cN} 0$ with negative AUS or US guided FNA, and planned BCS. Patients with clinically positive nodes, bilateral breast cancer, metastatic disease, history of invasive breast cancer, previous axillary surgery or RT, pregnant or nursing, and prior malignancies within past 5 years are excluded. Primary outcome is a regional recurrence rate (up to 10 years). Unlike the SOUND and INSEMA trials, this trial will include patients who have received neoadjuvant chemotherapy.

\section{Future Staging Algorithms}

The completion of the SOUND, INSEMA, and BOOG 2013-08 trials will likely alter staging algorithms for T12 breast cancer with axillary ultrasound playing a central role. Evaluation of newly diagnosed T1-2 breast cancer will first undergo AUS (Figure 4). Patients with no suspicious findings are spared from additional axillary staging procedures and are eligible for observation. Those with suspicious findings will undergo ultrasound-guided FNA or core biopsy. If ultrasound-guided biopsy is positive, patient will proceed with the standard surgical staging algorithm of SLNB with our without completion ALND. Patients with a negative biopsy may be safely observed.

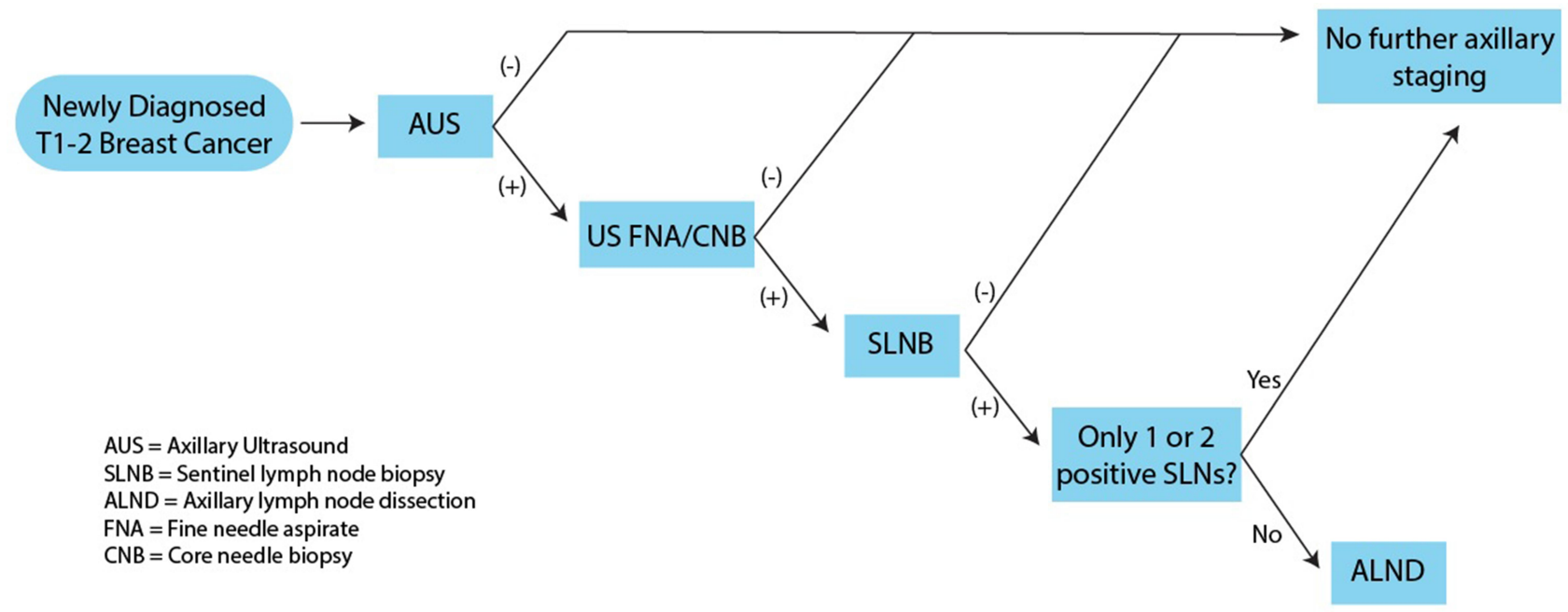

Figure 4 Possible future algorithm for staging of the axilla in early stage breast cancer. Several studies are currently ongoing evaluating the ability of AUS to accurately exclude disease in the axilla. If these studies are successful, the paradigm for axillary staging will evolve. Patients with newly diagnosed TI-2 breast cancer will be evaluated with an AUS. Patients with negative AUS will not require further axillary staging. Patients with a positive AUS will undergo FNA/CNB. Patients with a negative FNA/CNB can be observed. Patients with a positive FNA/CNB should undergo SLNB, possible ALND. 


\section{New Applications and Technologies} Contrast Enhanced Ultrasound

Contrast enhanced ultrasound (CEUS) is a relatively new technique for imaging of the axilla introduced by Omoto et al. ${ }^{60}$ The current technique involves an intradermal injection of $1 \mathrm{cc}$ of $25 \%$ albumin solution as contrast in the upper outer quadrant of the breast adjacent to the nipple. The breast is then massaged to encourage uptake of microbubbles into the lymphatic system. Using contrast pulse sequencing, microbubbles are followed in real-time to the axilla. The first axillary lymph node to be filled by contrast is assumed to be the sentinel node. ${ }^{61}$

Xie et al described three types of lymph node enhancement: type I, homogenously enhanced; type II, heterogenous enhancement; type III, weakly enhancing or nonenhancing. ${ }^{62}$ When type I nodes were considered as negative and type II \& III nodes as positive, the authors found ultrasound to have a sensitivity and specificity of $86.2 \%$ and $84.7 \%$, respectively, for detecting metastatic lymph nodes.

Current methods for identification of the sentinel lymph node during surgery include intraoperative use of lymphazurin/methylene blue dye and radiolabeled colloid. ${ }^{63}$ CEUS has been investigated as an alternative in identifying the target node. Comparing CEUS to methylene blue, Xie et al found no difference in correct identification of the sentinel lymph node between the two techniques. ${ }^{62}$ In a prospective case series of patients who underwent pre-operative CEUS-guided core needle biopsies followed by surgical staging, CEUS correctly identified the sentinel node in 555 out of 605 cases. ${ }^{64}$

Another technique uses a dual-modality photoacoustic and ultrasound imaging system to directly identify methylene blue accumulation in lymph nodes. Uribe et al demonstrated this concept in a pilot study of 16 patients. The goal of these technologies is to eventually perform percutaneous sentinel node biopsies using these technologies as detailed below. ${ }^{65}$

\section{Ultrasound-Guided Biopsy of the Sentinel Node}

Accurate identification of the sentinel lymph node offers the opportunity for percutaneous biopsy, which avoids the morbidity of an SLNB. In a meta-analysis, ultrasoundguided FNA and CNB had sensitivity of $74 \%$ and $88 \%$, respectively, for diagnosing nodal metastases, and both had specificity of $100 \% .{ }^{66}$ Some have proposed that
CEUS-guided SLN-FNA can effectively replace SLNB in identifying patients requiring ALND, but there may be higher rate of false-negative results in HER2-negative patients. ${ }^{67,68}$ When used as a pre-operative screening tool, Deurloo et al showed a $14 \%$ decrease in the number of SLNBs required. ${ }^{69}$ However, some evidence suggests that in patients meeting ACOSOG Z0011 criteria, using US-guided FNA as pre-operative triage may lead to overtreatment. ${ }^{70}$

\section{Prediction Nomograms}

Nomograms that incorporate ultrasound findings with tumor characteristics have been developed to predict the presence of lymph node metastasis. Qiu et al created the Shantou Nomogram assigning points to sonographic factors, such as lymph node diameter, cortical thickness, and fatty hilum loss, and tumor-related factors, including tumor size, histologic grade, and estrogen receptor status. ${ }^{71}$ The AUC was 0.9375 and 0.864 in the modeling group and validation group, respectively, confirming that nomograms may improve the ability of AUS in patient selection for axillary staging procedures. Tran et al showed that SLNB may be selectively omitted in patients with low probability of nodal metastasis based on the Shantou nomogram if used as a pre-operative screen. ${ }^{72}$

Intraoperatively, a nomogram could be used to predict the presence of lymph node metastasis using ex vivo shear wave elastography (SWE), an ultrasound modality. ${ }^{73}$ In a cohort 55 patients, excised lymph nodes were first examined using SWE and subsequently sent for pathological analysis. AUC was 0.856 and 0.791 in the development and validation cohorts, respectively.

\section{Targeted Axillary Lymph Node Dissection}

Post-NAC, SLNB has been shown to have lower yield and higher false-positive rates. ${ }^{74-76}$ Targeted axillary lymph node dissection (TAD) has been proposed, which involves the post-NAC removal of a node with documented metastasis on pre-NAC imaging. ${ }^{77}$ Ultrasound is used to mark diseased nodes pre-NAC with clips, ink, radiofrequency tags, or radioactive or magnetic seeds. The various techniques and their pros and cons are reviewed extensively by Banys-Paluchowski et al and clinical trials are currently underway to investigate their utility in clinical practice. ${ }^{78}$

\section{Radiomics}

Radiomics is the process of converting radiologic images into high dimensional mineable data. After volume of 
interest (VOI) is selected, extraction algorithms are used to generate datasets based on radiomic features (eg intensity, shape, texture, or wavelet). ${ }^{79}$ Several nomograms have been created using ultrasound radiomics and LASSO regression to predict the presence of lymph node metastasis with high AUCs. ${ }^{80,81}$ With the application of deep learning to ultrasound radiomics datasets, known as deep learning radiomics ultrasonography (DLRU), axillary lymph node status may be accurately predicted with AUC of $>0.90 .{ }^{82,83}$ Although radiomics is still in its infancy, it has great potential to pave the way for the personalization of surgical management of the axilla and "virtual biopsies."

\section{Conclusion}

\section{Paradigm Shift from Anatomic Staging to Tumor Biology}

Axillary surgery in breast cancer has undergone a dramatic change - from axillary lymph node dissection to the use of sentinel lymph node biopsy, reserving full dissection only for select patients. Historically, axillary surgery offered important anatomic staging information and was believed to be therapeutic by removing what was considered to be an important nidus of future metastasis. However, medical decision-making is increasingly driven by data derived from the primary tumor such as biomarker and molecular profile. ${ }^{53,54}$ Furthermore, new information about the biology of tumor metastasis suggests that metastatic disease typically originates in the primary tumor rather than in metastatic lymph nodes. $^{12-14}$ The ACOSOG Z0011 trial provides strong evidence that axillary surgery is no longer therapeutic, demonstrating that patients with early-stage breast cancer and 1-2 positive sentinel lymph nodes did not benefit from further axillary lymph node dissection. Taken together, evolving paradigms in breast cancer management have called into question the necessity of surgical axillary staging, and have mirrored broader changes in oncology to more personalized therapy informed by biomarkers. ${ }^{84-86}$

\section{Axillary Ultrasound in New Staging Algorithm}

Axillary ultrasound is an attractive modality to continue the de-escalation of the management of the axilla in breast cancer. Surgical staging is invasive for patients, and represents a significant economic cost for the healthcare system. Staging with AUS in lieu of surgical staging has demonstrated increased QALYs and monetary savings. ${ }^{51}$ Three multicenter prospective randomized clinical trials, the SOUND, INSEMA, and BOOG 2013-08, are underway to investigate the potential of using axillary ultrasound to stage early breast cancer. These trials will provide valuable information in constructing future staging algorithms where AUS will play a central role, potentially replacing surgical staging. Patients will benefit from avoidance of an invasive surgery, while the healthcare system will be relieved of an economic burden.

\section{Outlook}

As AUS play a more central role in staging of the axilla, new technologies are on the horizon with the potential to increase its utility and expand its role. Biopsies guided by contrast enhanced ultrasound represent another potential alternative to SLNB. Prediction nomograms are simple tools that synthesize clinical, biological, and sonographic features to predict axillary disease burden and aid surgical decision making. ${ }^{71,72}$ The budding field of radiomics applies neural networks and deep learning to ultrasound imaging to construct models not possible previously. ${ }^{87}$ These developments could allow AUS to be incorporated into the treatment of advanced stage cancers beyond those included in the Z0011 trial. The surgical management of breast cancer has undergone many transformations over the past century. Axillary ultrasound represents the next phase in this radical transformation, and is certain to play a central role in future staging algorithms of the axilla in breast cancer.

\section{Author Contributions}

Michael Y Chen, MD, William E. Gillanders, MD

All authors made substantial contributions to conception and design, acquisition of data, or analysis and interpretation of data; took part in drafting the article or revising it critically for important intellectual content; agreed to submit to the current journal; gave final approval for the version to be published; and agreed to be accountable for all aspects of the work.

\section{Funding}

This publication was supported by the Washington University School of Medicine Surgical Oncology Basic Science and Translational Research Training Program grant T32CA009621. The content is solely the responsibility of the authors and does not necessarily represent the official views of the NIH. 


\section{Disclosure}

The authors report no conflicts of interest in this work.

\section{References}

1. Siegel RL, Miller KD, Fuchs HE, Jemal A. Cancer statistics, 2021. CA Cancer J Clin. 2021;71(1):7-33. doi:10.3322/caac.21654

2. Magnoni F, Galimberti V, Corso G, Intra M, Sacchini V, Veronesi P. Axillary surgery in breast cancer: an updated historical perspective. Semin Oncol. 2020;47(6):341-352. doi:10.1053/j. seminoncol.2020.09.001

3. Halsted WS. The results of radical operations for the cure of carcinoma of the breast. Ann Surg. 1907;46(1):1. doi:10.1097/00000658190707000-00001

4. Freeman MD, Gopman JM, Salzberg CA. The evolution of mastectomy surgical technique: from mutilation to medicine. Gland Surg. 2018;7(3):308-315. doi:10.21037/gs.2017.09.07

5. Lucci A, McCall LM, Beitsch PD, et al. Surgical complications associated with sentinel lymph node dissection (SLND) plus axillary lymph node dissection compared with SLND alone in the American College of Surgeons Oncology Group trial Z0011. J Clin Oncol. 2007;25(24):3657-3663. doi:10.1200/JCO.2006.07.4062

6. Fleissig A, Fallowfield LJ, Langridge CI, et al. Post-operative arm morbidity and quality of life. Results of the ALMANAC randomised trial comparing sentinel node biopsy with standard axillary treatment in the management of patients with early breast cancer. Breast Cancer Res Treat. 2006;95(3):279-293. doi:10.1007/s10549-005-9025-7

7. Giuliano AE, Edge SB, Hortobagyi GN. of the AJCC cancer staging manual: breast cancer. Ann Surg Oncol. 2018;25(7):1783-1785. doi:10.1245/s10434-018-6486-6

8. National Comprehensive Cancer Network. Invasive breast cancer (Version 6.2020). Available from: https://www.nccn.org/profes sionals/physician_gls/pdf/breast.pdf. Accessed November 12, 2020.

9. Sørlie T, Perou CM, Tibshirani R, et al. Gene expression patterns of breast carcinomas distinguish tumor subclasses with clinical implications. Proc Natl Acad Sci U S A. 2001;98(19):10869-10874. doi:10.1073/pnas.191367098

10. Perou CM, Sørlie T, Eisen MB, et al. Molecular portraits of human breast tumours. Nature. 2000;406(6797):747-752. doi:10.1038/ 35021093

11. Yachida S, Jones S, Bozic I, et al. Distant metastasis occurs late during the genetic evolution of pancreatic cancer. Nature. 2010;467 (7319):1114-1117. doi:10.1038/nature09515

12. Engel J, Emeny RT, Hölzel D. Positive lymph nodes do not metastasize. Cancer Metastasis Rev. 2012;31(1-2):235-246. doi:10.1007/s10555-011-9343-7

13. Ullah I, Karthik GM, Alkodsi A, et al. Evolutionary history of metastatic breast cancer reveals minimal seeding from axillary lymph nodes. $J$ Clin Invest. 2018;128(4):1355-1370. doi:10.1172/JCI96149

14. Lambert AW, Pattabiraman DR, Weinberg RA. Emerging biological principles of metastasis. Cell. 2017;168(4):670-691. doi:10.1016/j. cell.2016.11.037

15. Giuliano AE, Ballman KV, McCall L, et al. Effect of axillary dissection vs no axillary dissection on 10-year overall survival among women with invasive breast cancer and sentinel node metastasis: the ACOSOG Z0011 (Alliance) Randomized Clinical Trial. JAMA. 2017;318(10):918-926. doi:10.1001/jama.2017.11470

16. Galimberti V, Cole BF, Zurrida S, et al. Axillary dissection versus no axillary dissection in patients with sentinel-node micrometastases (IBCSG 23-01): a Phase 3 randomised controlled trial. Lancet Oncol. 2013;14(4):297-305. doi:10.1016/S1470-2045(13)70035-4
17. Giuliano AE, McCall L, Beitsch $\mathrm{P}$, et al. Locoregional recurrence after sentinel lymph node dissection with or without axillary dissection in patients with sentinel lymph node metastases: the American College of Surgeons Oncology Group Z0011 randomized trial. Ann Surg. 2010;252(3):426-432; discussion 432-423. doi:10.1097/ SLA.0b013e3181f08f32

18. Giuliano AE, Hunt KK, Ballman KV, et al. Axillary dissection vs no axillary dissection in women with invasive breast cancer and sentinel node metastasis: a randomized clinical trial. JAMA. 2011;305 (6):569-575. doi:10.1001/jama.2011.90

19. Burstein HJ, Curigliano G, Loibl S, et al. Estimating the benefits of therapy for early-stage breast cancer: the St. Gallen International Consensus Guidelines for the primary therapy of early breast cancer 2019. Ann Oncol. 2019;30(10):1541-1557. doi:10.1093/annonc/mdz235

20. de Freitas R Jr, Costa MV, Schneider SV, Nicolau MA, Marussi E. Accuracy of ultrasound and clinical examination in the diagnosis of axillary lymph node metastases in breast cancer. Eur J Surg Oncol. 1991;17(3):240-244.

21. Lanng C, Hoffmann J, Galatius H, Engel U. Assessment of clinical palpation of the axilla as a criterion for performing the sentinel node procedure in breast cancer. Eur J Surg Oncol. 2007;33(3):281-284. doi:10.1016/j.ejso.2006.09.032

22. Vaidya JS, Vyas JJ, Thakur MH, Khandelwal KC, Mittra I. Role of ultrasonography to detect axillary node involvement in operable breast cancer. Eur J Surg Oncol. 1996;22(2):140-143. doi:10.1016/ S0748-7983(96)90593-4

23. Dialani V, James DF, Slanetz PJ. A practical approach to imaging the axilla. Insights Imaging. 2015;6(2):217-229. doi:10.1007/s13244014-0367-8

24. Tucker NS, Cyr AE, Ademuyiwa FO, et al. Axillary ultrasound accurately excludes clinically significant lymph node disease in patients with early stage breast cancer. Ann Surg. 2016;264 (6):1098-1102. doi:10.1097/SLA.0000000000001549

25. Veronesi U, Paganelli G, Viale G, et al. Sentinel-lymph-node biopsy as a staging procedure in breast cancer: update of a randomised controlled study. Lancet Oncol. 2006;7(12):983-990. doi:10.1016/ S1470-2045(06)70947-0

26. Krag DN, Anderson SJ, Julian TB, et al. Sentinel-lymph-node resection compared with conventional axillary-lymph-node dissection in clinically node-negative patients with breast cancer: overall survival findings from the NSABP B-32 randomised phase 3 trial. Lancet Oncol. 2010;11(10):927-933. doi:10.1016/S1470-2045(10)70207-2

27. Mansel RE, Fallowfield L, Kissin M, et al. Randomized multicenter trial of sentinel node biopsy versus standard axillary treatment in operable breast cancer: the ALMANAC Trial. J Natl Cancer Inst. 2006;98(9):599-609. doi:10.1093/jnci/djj158

28. Ashikaga T, Krag DN, Land SR, et al. Morbidity results from the NSABP B-32 trial comparing sentinel lymph node dissection versus axillary dissection. J Surg Oncol. 2010;102(2):111-118. doi:10.1002/ jso. 21535

29. Kim T, Giuliano AE, Lyman GH. Lymphatic mapping and sentinel lymph node biopsy in early-stage breast carcinoma: a metaanalysis. Cancer. 2006;106(1):4-16. doi:10.1002/cncr.21568

30. Lyman GH, Giuliano AE, Somerfield MR, et al. American Society of Clinical Oncology guideline recommendations for sentinel lymph node biopsy in early-stage breast cancer. J Clin Oncol. 2005;23 (30):7703-7720. doi:10.1200/JCO.2005.08.001

31. Gooch J, King TA, Eaton A, et al. The extent of extracapsular extension may influence the need for axillary lymph node dissection in patients with T1-T2 breast cancer. Ann Surg Oncol. 2014;21 (9):2897-2903. doi:10.1245/s10434-014-3752-0

32. Amin MB, Edge SB. AJCC Cancer Staging Manual. Springer; 2017. 
33. Mustonen P, Farin P, Kosunen O. Ultrasonographic detection of metastatic axillary lymph nodes in breast cancer. Ann Chir Gynaecol. 1990;79(1):15-18.

34. Tate JJ, Lewis V, Archer T, Guyer PG, Royle GT, Taylor I. Ultrasound detection of axillary lymph node metastases in breast cancer. Eur J Surg Oncol. 1989;15(2):139-141.

35. Bedi DG, Krishnamurthy R, Krishnamurthy S, et al. Cortical morphologic features of axillary lymph nodes as a predictor of metastasis in breast cancer: in vitro sonographic study. AJR Am J Roentgenol. 2008;191(3):646-652. doi:10.2214/AJR.07.2460

36. Mainiero MB, Cinelli CM, Koelliker SL, Graves TA, Chung MA. Axillary ultrasound and fine-needle aspiration in the preoperative evaluation of the breast cancer patient: an algorithm based on tumor size and lymph node appearance. AJR Am J Roentgenol. 2010;195(5):1261-1267. doi:10.2214/AJR.10.4414

37. Net JM, Mirpuri TM, Plaza MJ, et al. Resident and fellow education feature: US evaluation of axillary lymph nodes. Radiographics. 2014;34(7):1817-1818. doi:10.1148/rg.347140081

38. Kim WH, Kim HJ, Lee SM, et al. Preoperative axillary nodal staging with ultrasound and magnetic resonance imaging: predictive values of quantitative and semantic features. $\mathrm{Br} J$ Radiol. 2018;91 (1092):20180507. doi:10.1259/bjr.20180507

39. Zhang H, Sui X, Zhou S, Hu L, Huang X. Correlation of conventional ultrasound characteristics of breast tumors with axillary lymph node metastasis and $\mathrm{Ki}-67$ expression in patients with breast cancer. J Ultrasound Med. 2019;38(7):1833-1840. doi:10.1002/jum.14879

40. Houssami N, Ciatto S, Turner RM, Cody HS 3rd, Macaskill P. Preoperative ultrasound-guided needle biopsy of axillary nodes in invasive breast cancer: meta-analysis of its accuracy and utility in staging the axilla. Ann Surg. 2011;254(2):243-251. doi:10.1097/ SLA.0b013e31821f1564

41. Verheuvel NC, van den Hoven I, Ooms HW, Voogd AC, Roumen RM. The role of ultrasound-guided lymph node biopsy in axillary staging of invasive breast cancer in the post-ACOSOG Z0011 Trial Era. Ann Surg Oncol. 2014. doi:10.1245/s10434-0144071-1

42. Caudle AS, Kuerer HM, Le-petross HT, et al. Predicting the extent of nodal disease in early-stage breast cancer. Ann Surg Oncol. 2014;21 (11):3440-3447. doi:10.1245/s10434-014-3813-4

43. Hieken TJ, Trull BC, Boughey JC, et al. Preoperative axillary imaging with percutaneous lymph node biopsy is valuable in the contemporary management of patients with breast cancer. Surgery. 2013;154(4):831-838; discussion 838-840. doi:10.1016/j.surg.2013.07.017

44. Joh JE, Han G, Kiluk JV, Laronga C, Khakpour N, Lee MC. Indications for axillary ultrasound use in breast cancer patients. Clin Breast Cancer. 2012;12(6):433-437. doi:10.1016/j.clbc.2012.09.009

45. Boughey JC, Ballman KV, Hunt KK, et al. Axillary ultrasound after neoadjuvant chemotherapy and its impact on sentinel lymph node surgery: results from the American College of Surgeons Oncology Group Z1071 Trial (Alliance). J Clin Oncol. 2015;33(30):3386-3393. doi:10.1200/JCO.2014.57.8401

46. Hieken TJ, Boughey JC, Jones KN, Shah SS, Glazebrook KN. Imaging response and residual metastatic axillary lymph node disease after neoadjuvant chemotherapy for primary breast cancer. Ann Surg Oncol. 2013;20(10):3199-3204. doi:10.1245/ s10434-013-3118-z

47. Di Micco R, Zuber V, Fiacco E, et al. Sentinel node biopsy after primary systemic therapy in node positive breast cancer patients: time trend, imaging staging power and nodal downstaging according to molecular subtype. Eur J Surg Oncol. 2019;45(6):969-975. doi:10.1016/j.ejso.2019.01.219
48. Wilke LG, McCall LM, Posther KE, et al. Surgical complications associated with sentinel lymph node biopsy: results from a prospective international cooperative group trial. Ann Surg Oncol. 2006;13(4):491-500. doi:10.1245/ASO.2006.05.013

49. Land SR, Kopec JA, Julian TB, et al. Patient-reported outcomes in sentinel node-negative adjuvant breast cancer patients receiving sentinel-node biopsy or axillary dissection: National Surgical Adjuvant Breast and Bowel Project Phase III protocol B-32. J Clin Oncol. 2010;28(25):3929-3936. doi:10.1200/JCO.2010.28.2491

50. Henry-Tillman R, Glover-Collins K, Preston M, et al. The SAVE review: sonographic analysis versus excision for axillary staging in breast cancer. J Am Coll Surg. 2015;220(4):560-567. doi:10.1016/j. jamcollsurg.2014.12.033

51. McEvoy AM, Poplack S, Nickel K, et al. Cost-effectiveness analyses demonstrate that observation is superior to sentinel lymph node biopsy for postmenopausal women with $\mathrm{HR}+$ breast cancer and negative axillary ultrasound. Breast Cancer Res Treat. 2020:1-12.

52. Ramsey SD, Barlow WE, Gonzalez-Angulo AM, et al. Integrating comparative effectiveness design elements and endpoints into a Phase III, randomized clinical trial (SWOG S1007) evaluating oncotypeDX-guided management for women with breast cancer involving lymph nodes. Contemp Clin Trials. 2013;34(1):1-9. doi:10.1016/j.cet.2012.09.003

53. Stemmer SM, Steiner M, Rizel S, et al. Clinical outcomes in ER+ HER2 -node-positive breast cancer patients who were treated according to the Recurrence Score results: evidence from a large prospectively designed registry. NPJ Breast Cancer. 2017;3:32. doi:10.1038/ s41523-017-0033-7

54. Sparano JA, Gray RJ, Makower DF, et al. Adjuvant chemotherapy guided by a 21-gene expression assay in breast cancer. $N$ Engl J Med. 2018;379(2):111-121. doi:10.1056/NEJMoa1804710

55. Sparano JA, Gray RJ, Makower DF, et al. Prospective validation of a 21-gene expression assay in breast cancer. $N$ Engl J Med. 2015;373 (21):2005-2014. doi:10.1056/NEJMoa1510764

56. Vicini FA, Horwitz EM, Lacerna MD, et al. The role of regional nodal irradiation in the management of patients with early-stage breast cancer treated with breast-conserving therapy. Int J Radiat Oncol Biol Phys. 1997;39(5):1069-1076. doi:10.1016/S0360-3016(97)00555-5

57. Whelan TJ, Olivotto IA, Parulekar WR, et al. Regional nodal irradiation in early-stage breast cancer. $N$ Engl $J$ Med. 2015;373 (4):307-316. doi:10.1056/NEJMoa1415340

58. Hirata K, Yoshimura M, Inoue $\mathrm{M}$, et al. Regional recurrence in breast cancer patients with one to three positive axillary lymph nodes treated with breast-conserving surgery and whole breast irradiation. J Radiat Res. 2017;58(1):79-85. doi:10.1093/jrr/rrw071

59. Cyr AE, Tucker N, Ademuyiwa F, et al. Successful completion of the pilot phase of a randomized controlled trial comparing sentinel lymph node biopsy to no further axillary staging in patients with clinical T1-T2 N0 breast cancer and normal axillary ultrasound. $J$ Am Coll Surg. 2016;223(2):399-407. doi:10.1016/j.jamcollsurg.2016.04.048

60. Omoto K, Hozumi Y, Omoto Y, et al. Sentinel node detection in breast cancer using contrast-enhanced sonography with $25 \%$ albumin -Initial clinical experience. J Clin Ultrasound. 2006;34(7):317-326. doi:10.1002/jcu.20241

61. Sharma N, Cox K. Axillary nodal staging with contrast-enhanced ultrasound. Curr Breast Cancer Rep. 2017;9(4):259-263. doi:10.1007/s12609-017-0258-3

62. Xie F, Zhang D, Cheng L, et al. Intradermal microbubbles and contrast-enhanced ultrasound (CEUS) is a feasible approach for sentinel lymph node identification in early-stage breast cancer. World J Surg Oncol. 2015;13:319. doi:10.1186/s12957-015-0736-x 
63. Albertini JJ, Lyman GH, Cox C, et al. Lymphatic mapping and sentinel node biopsy in the patient with breast cancer. JAMA. 1996;276(22):1818-1822.

64. Cox K, Weeks J, Mills P, et al. Contrast-enhanced ultrasound biopsy of sentinel lymph nodes in patients with breast cancer: implications for axillary metastases and conservation. Ann Surg Oncol. 2016;23 (1):58-64. doi:10.1245/s10434-015-4606-0

65. Garcia-Uribe A, Erpelding TN, Krumholz A, et al. Dual-modality photoacoustic and ultrasound imaging system for noninvasive sentinel lymph node detection in patients with breast cancer. Sci Rep. 2015;5:15748. doi:10.1038/srep15748

66. Balasubramanian I, Fleming CA, Corrigan MA, Redmond HP, Kerin MJ, Lowery AJ. Meta-analysis of the diagnostic accuracy of ultrasound-guided fine-needle aspiration and core needle biopsy in diagnosing axillary lymph node metastasis. Br J Surg. 2018;105 (10):1244-1253. doi:10.1002/bjs. 10920

67. Zhong J, Sun DS, Wei W, et al. Contrast-enhanced ultrasound-guided fine-needle aspiration for sentinel lymph node biopsy in early-stage breast cancer. Ultrasound Med Biol. 2018;44(7):1371-1378. doi:10.1016/j.ultrasmedbio.2018.03.005

68. Rukanskienė D, Veikutis V, Jonaitienè E, et al. Preoperative axillary ultrasound versus sentinel lymph node biopsy in patients with early breast cancer. Medicina. 2020;56(3). doi:10.3390/ medicina56030127

69. Deurloo EE, Tanis PJ, Gilhuijs KGA, et al. Reduction in the number of sentinel lymph node procedures by preoperative ultrasonography of the axilla in breast cancer. Eur J Cancer. 2003;39(8):1068-1073. doi:10.1016/S0959-8049(02)00748-7

70. Del Riego J, Diaz-Ruiz MJ, Teixidó M, et al. The impact of axillary ultrasound with biopsy in overtreatment of early breast cancer. Eur J Radiol. 2018;98:158-164. doi:10.1016/j.ejrad.2017.11.018

71. Qiu S-Q, Zeng H-C, Zhang F, et al. A nomogram to predict the probability of axillary lymph node metastasis in early breast cancer patients with positive axillary ultrasound. Sci Rep. 2016;6(1):1-12.

72. Tran HT, Pack D, Mylander C, et al. Ultrasound-based nomogram identifies breast cancer patients unlikely to harbor axillary metastasis: towards selective omission of sentinel lymph node biopsy. Ann Surg Oncol. 2020;27(8):2679-2686. doi:10.1245/s10434-019-08164-3

73. Bae SJ, Youk JH, Yoon CI, et al. A nomogram constructed using intraoperative ex vivo shear-wave elastography precisely predicts metastasis of sentinel lymph nodes in breast cancer. Eur Radiol. 2020;30(2):789-797. doi:10.1007/s00330-019-06473-5

74. Boughey JC, Suman VJ, Mittendorf EA, et al. Sentinel lymph node surgery after neoadjuvant chemotherapy in patients with node-positive breast cancer: the ACOSOG Z1071 (Alliance) Clinical Trial. JAMA. 2013;310(14):1455-1461. doi:10.1001/jama.2013.278932

75. Kuehn T, Bauerfeind I, Fehm T, et al. Sentinel-lymph-node biopsy in patients with breast cancer before and after neoadjuvant chemotherapy (SENTINA): a prospective, multicentre cohort study. Lancet Oncol. 2013;14(7):609-618. doi:10.1016/S1470-2045(13)70166-9
76. Boileau J-F, Poirier B, Basik M, et al. Sentinel node biopsy after neoadjuvant chemotherapy in biopsy-proven node-positive breast cancer: the SN FNAC study. J Clin Oncol. 2015;33(3):258-264. doi:10.1200/JCO.2014.55.7827

77. Caudle AS, Yang WT, Krishnamurthy S, et al. Improved axillary evaluation following neoadjuvant therapy for patients with node-positive breast cancer using selective evaluation of clipped nodes: implementation of targeted axillary dissection. J Clin Oncol. 2016;34(10):1072. doi:10.1200/JCO.2015.64.0094

78. Banys-Paluchowski M, Gruber IV, Hartkopf A, et al. Axillary ultrasound for prediction of response to neoadjuvant therapy in the context of surgical strategies to axillary dissection in primary breast cancer: a systematic review of the current literature. Arch Gynecol Obstet. 2020;301(2):341-353. doi:10.1007/s00404-019-05428-x

79. Lambin P, Leijenaar RTH, Deist TM, et al. Radiomics: the bridge between medical imaging and personalized medicine. Nat Rev Clin Oncol. 2017;14(12):749-762. doi:10.1038/nrclinonc.2017.141

80. Yu F-H, Wang J-X, Ye X-H, Deng J, Hang J, Yang B. Ultrasoundbased radiomics nomogram: a potential biomarker to predict axillary lymph node metastasis in early-stage invasive breast cancer. Eur J Radiol. 2019;119:108658. doi:10.1016/j.ejrad.2019.108658

81. Gao Y, Luo Y, Zhao C, et al. Nomogram based on radiomics analysis of primary breast cancer ultrasound images: prediction of axillary lymph node tumor burden in patients. Eur Radiol. 2020.

82. Zheng X, Yao Z, Huang Y, et al. Deep learning radiomics can predict axillary lymph node status in early-stage breast cancer. Nat Commun. 2020;11(1):1236. doi:10.1038/s41467-020-15027-z

83. Guo X, Liu Z, Sun C, et al. Deep learning radiomics of ultrasonography: identifying the risk of axillary non-sentinel lymph node involvement in primary breast cancer. EBioMedicine. 2020;60:103018. doi:10.1016/j.ebiom.2020.103018

84. Crigna AT, Samec M, Koklesova L, et al. Cell-free nucleic acid patterns in disease prediction and monitoring-hype or hope? EPMA J. 2020;11(4):1-25.

85. Qian S, Golubnitschaja O, Zhan X. Chronic inflammation: key player and biomarker-set to predict and prevent cancer development and progression based on individualized patient profiles. EPMA J. 2019;10(4):365-381. doi:10.1007/s13167-019-00194-x

86. Samec M, Liskova A, Koklesova L, et al. Flavonoids against the Warburg phenotype-concepts of predictive, preventive and personalised medicine to cut the Gordian knot of cancer cell metabolism. EPMA J. 2020;11(3):377-398. doi:10.1007/s13167-020-00217-y

87. Guo Q, Dong Z, Zhang L, et al. Ultrasound features of breast cancer for predicting axillary lymph node metastasis. $J$ Ultrasound Med. 2018;37(6):1353-1354. doi:10.1002/jum.14469
Breast Cancer: Targets and Therapy

\section{Publish your work in this journal}

Breast Cancer - Targets and Therapy is an international, peer-reviewed open access journal focusing on breast cancer research, identification of therapeutic targets and the optimal use of preventative and integrated treatment interventions to achieve improved outcomes, enhanced survival and quality of life for the cancer patient.
The manuscript management system is completely online and includes a very quick and fair peer-review system, which is all easy to use. Visit http://www.dovepress.com/testimonials.php to read real quotes from published authors. 